\title{
Case Report on Bipolar Affective Disorder: Mania with Psychotic Symptoms
}

\author{
Kounassegarane Deepika
}

\begin{abstract}
Bipolar affective disorder (BPAD) is a major psychiatric disorder all around the world, which is mainly characterized by frequent and recurrent episodes of mania, hypomania, and depression. A majority of complete etiology or pathogenesis of BPAD is unknown. Mania occurs for a period of 1 week or more where the affected individual may experience a change in normal behavior that drastically affects their normal functioning. The defining characteristics of mania are alteration in mood (elation and ecstasy), increased talkativeness, rapid speech, sleep disturbance, racing thoughts, increase in their goal-directed activity, increased psychomotor activity, and poor insight. Some other major signs and symptoms of mania are an elevated or expansive mood, mood changes, impulsive behavior, irritability, and grandiose ideas. The manic episode usually presents with psychotic features, which may include delusions and hallucinations. Those persons are highly intended to respond to outsiders for their psychosis as well as for their manic episode, which is mainly noticed by others, including each member of the family, friends, and even strangers. Rapid cycling of the bipolar disorder is defined as the individual may present with at least four or more episodes of mood changes for a period of 12 months.

Keywords: Bipolar disorder, Delusion, Ecstasy, Elation, Hallucination, Mania, Psychosis.

Pondicherry Journal of Nursing (2019): 10.5005/jp-journals-10084-12115
\end{abstract}

\section{INTRODUCTION}

Mania occurs for a period of 1 week or more where the affected individual may experience a change in normal behavior that drastically affects their normal functioning. ${ }^{1}$ The defining characteristics of mania are alteration in mood (elation and ecstasy), increased talkativeness, rapid speech, sleep disturbance, racing thoughts, increase in their goal-directed activity, increased psychomotor activity, and poor insight. ${ }^{2}$

\section{Case Description}

A 16-year-old female got admitted with the complaints of decreased sleep, irritable and excessively happy, irrelevant and increased speech, use of abusing words to her parents, and had a grandiose idea that she is having a power of Lord Shiva for past 4 days. Her onset of symptoms is sudden with 6 months of duration. She had a predisposing factor as family history of the bipolar affective disorder for her mother and now she is under the treatment. She was an introvert person, she isolated herself, and she did not maintain a good relationship with her family members and friends. She had a fear of getting less marks in her 11th board examination. During assessment her vitals were stable, and she had a previous history of catatonia for which she received treatment in MGMCRI in March 2019 and was advised to have a regular follow-up. She was not under regular treatment and follow-up, and then she developed the symptoms of feeling sad, not interacting with her family members, and not able to maintain her activities of daily living (ADL). There she was under the treatment for 2 weeks, and the physician advised to continue treatment for at least 3-4 months. But she took medicines only for 2 weeks and as she felt normal she stopped taking drugs; due to noncompliance, then she developed the above symptoms. Then she was brought to MGMCRI for the further management; there she underwent investigations such as history collection, mental status examination, etc., and based on the ICD 10 criteria she was diagnosed as having the bipolar affective disorder. She was
Department of Mental Health Nursing, Kasturba Gandhi Nursing College, Sri Balaji Vidyapeeth Deemed University, Puducherry, India

Corresponding Author: Kounassegarane Deepika, Department of Mental Health Nursing, Kasturba Gandhi Nursing College, Sri Balaji Vidyapeeth Deemed University, Puducherry, India, Phone: +91 9629750987, e-mail: deepsdeeps7287@gmail.com

How to cite this article: Deepika K. Case Report on Bipolar Affective Disorder: Mania with Psychotic Symptoms. Pon J Nurs 2019;12(2): 50-51.

Source of support: Nil

Conflict of interest: None

under pharmacotherapy and psychotherapy, which improved her condition and she was discharged.

\section{History Taking}

During history taking, the following findings were noted: in her childhood she was an introvert person, she isolated herself, she did not maintain a good relationship with her family members and friends and teachers, she had a fear of getting less marks in her 11th board examination, and she had noncompliance of drugs.

\section{Physical and Mental Status Examination}

In physical examination, vitals were stable. She had associated disturbances such as sleeping disturbance and loss of appetite. In mental status examination, the following findings were noted: increased psychomotor activity, delusion of grandiose (she said that she is having a super power of Lord Shiva), excessive talkativeness, mood elevation (shifting her thoughts from one topic to another), more strong in her speech, too difficult to interrupt, poor judgment, not able to concentrate in her daily routines, and poor insight (fourth-degree insight). 


\section{INVESTIGATIONS}

Blood investigation findings showed: serum creatinine $-0.75 \mathrm{mg} / \mathrm{dL}$, serum urea-15 $\mathrm{mg} / \mathrm{dL}$, serum sodium-142 mEq/dL, serum potassium - $5.1 \mathrm{mEq} / \mathrm{dL}$, and serum chloride-101 mEq/dL.

She underwent special investigation such as psychometric assessment-young mania rating scale (YMRS), and the findings had been recorded as score 37 and was identified as the manic episode.

Based on ICD 10 classification:

The patient was diagnosed as the $\mathrm{F} 30$ manic episode.

Other classification of mania may include:

F31 bipolar affective disorder; F31.0 bipolar affective disorder, current episode hypomanic; F31.1 bipolar affective disorder, current episode manic without psychotic symptoms; F31.2 bipolar affective disorder, current episode manic with psychotic symptoms; F31.3 bipolar affective disorder, current episode mild or moderate depression; F31.4 bipolar affective disorder, current episode severe depression without psychotic symptoms; F31.5 bipolar affective disorder, current episode severe depression with psychotic symptoms; F31.6 bipolar affective disorder, current episode mixed; F31.7 bipolar affective disorder, currently in remission; F31.8 other bipolar affective disorders; F31.9 bipolar affective disorder, unspecified.

\section{Treatment and Follow-up}

The client underwent treatment such as psychopharmacotherapy, electroconvulsive therapy (ECT), and other psychotherapies. Psychopharmacological therapy may include T. chlorpromazine 100 mg PO 0-0-2, T. lithium 300 mg PO 1-0-1, Syp. divalproex sodium $250 \mathrm{mg} / \mathrm{mL}$ PO $10 \mathrm{~mL}-0-10$ mL, Cap. pantop D1 cap PO (BF) 1-0-1, Syp. sucralfate $10 \mathrm{~mL}$ PO 1-1-1, and Cap. bifilac 1 cap PO 1-1-1. She underwent two sessions of ECT and there was no complications during the session; several other psychotherapies had been given such as individual and family counseling therapy, supportive therapy such as yoga and music therapy, and deep breathing techniques also had been taught to the patient. She got discharged after third session of ECT. She got improvement in her physical and psychological health, and then she got discharged and the family members were educated about drug noncompliance, availability of rehabilitation services, and follow-up services. She insisted for follow-up after 10 days.

\section{Differential Diagnosis}

- There are numerous differential diagnoses in the assessment of patients who present with symptoms like mania. Patients can be exhibiting numerous other physiological and psychiatric disorders. One of the most common situations that may mimic mania is caffeine or other stimulant intoxication, especially cocaine, amphetamine (including methamphetamine), $P C P$, and nicotine. ${ }^{3}$

- One of the most common problems is defining the disorder from schizophrenia, is particularly from the developmental stages through hypomania have been missed and the person is most likely at the peak of the illness with delusions, incongruent speech, and violent behavior may produce the basic disturbance in an affect.

- An individual with mania who is under the treatment with neuroleptic medication may present with a similar diagnostic problem at the stage when they have returned to normal levels of physical and mental activity but still may present with delusions and hallucinations. ${ }^{4}$

- Hallucinogens also produce mania-like symptoms. Excessive steroid and human growth hormone may cause aggression, irritability, and anxiety. ${ }^{3}$

- Personality disorders like histrionic and borderline personality may have similar symptoms of bipolar, which include mood changes, anger, inappropriate speech, and bizarre behavior. ${ }^{4}$

- Physiological conditions include hyperthyroidism, hypertensive urgency, hypercortisolemia, hyperaldosteronism, tumors and masses in the brain, major delirium, neurocognitive disorders, and acromegaly. ${ }^{4}$

\section{Discussion}

The prognosis of manic patients is favorable. Here the patient presented with many symptoms and after several therapies became normal because adhered to medications and all the therapies. Some factors associated with a poorer outcome are a history of abuse, psychosis, low socioeconomic status, comorbid illness, or a young age of the first onset. ${ }^{5}$

\section{References}

1. Berrios GE. Of mania. History of Psychiatry 2004;15(57 Pt 1):105-124. DOI: 10.1177/0957154X04041829.

2. Kramer M. ICD-10 Classification of Mental and Behavioural Disorders Clinical Descriptions and Diagnostic Guidelines. World Health Organization; 2012.

3. Khan O, Youssef NA. A brief history of polarity in mood and its diagnostic evolution. Ann Clin Psychiatry 2018;30(1):61-66.

4. Robins L. Diagnostic and statistical manual of mental disorders: DSM-5. 5th ed., Washington: American Psychiatric Publishing; 2013. pp. 237-242.

5. Davis S, Kenneth L. Neuropsychopharmacology. 5th ed., Philadelphia: Lippincott Williams \& Wilkins; 2002. pp. 1609-1610. 